\title{
Serotype Distribution and Antimicrobial Resistance of Streptococcus pneumoniae in the Philippines, 2004-2011*
}

Sonia Sia, Celia Carlos, Ma. Charmian Hufano, Marietta Lagrada, Joselle Ealdama, Ma. Theresa Sepulveda, Melissa Masim

Research Institute for Tropical Medicine - Department of Health, Philippines

\section{ABSTRACT}

Objective. Scarcity of data on the serotype composition and antibiotic resistance of invasive pneumococci from developing countries has been noted. ${ }^{13}$ We describe in this study the serogroup distribution and antimicrobial resistance patterns of Streptococcus pneumoniae in the Philippines from 2004-2011.

Methodology. S. pneumoniae isolated from patients with invasive pneumococcal disease (IPD) were referred to the Antimicrobial Resistance Surveillance Reference Laboratory from 2004 to 2011. Typing of isolates was done through slide agglutination and antimicrobial susceptibility was determined following CLSI methods.

Results. The penicillin-resistant meningitis isolates were of serotypes 1,5 and 14 which are covered by PCV10 and 13. The erythromycin resistant isolates were serotype 9 while cotrimoxazole resistant isolates were serotypes 1, 5, 6, 12 and 14. Forty-one percent of the cotrimoxazole resistant isolates are covered by PCV7, and $88 \%$ are covered by both PCV10 and PCV13. Levofloxacin resistant isolates were of serotypes 5 and 23 with PCV7 coverage of $50 \%$ and PCV 10 and PCV13 coverages of $100 \%$.

Conclusions. S. pneumoniae serotypes causing IPD in the country is largely similar to the dominant IPD serotypes worldwide. The serotype distribution in the Philippines remained stable from 2004 to 2011 and antimicrobial resistance among the isolates remained low. The serotypes of antibiotic resistant $S$. pneumoniae in this study were not similar with known serotype resistance profiles in other Asian countries. With the inclusion of PCV in the free national immunization program of the country beginning 2013, continued surveillance of prevailing pneumococcal serotypes should be done to monitor any shift in the prevalence of PCV associated serotypes to guide disease control measures including control of emergence of resistant pneumococcal isolates.

Key words: Streptococcus pneumoniae, antimicrobial resistance, serotype, ARSP

\section{ISSN 0118-3265}

Printed in the Philippines.

Copyright $\odot 2017$ by the PJP

Received: 7 March 2017.

Accepted: 6 April 2017.

Published online first: 9 April 2017.

https://doi.org/10.21141/PJP.2017.005

Corresponding author: Sonia B. Sia, MD

E-mail: ssiaphl@gmail.com

* Abstract was presented at the 10th International Symposium on Antimicrobial Agents and Resistance (ISAAR) 2015 from Mav 14 to 15, 2015 at Incheon, Korea

\section{INTRODUCTION}

Streptococcus pneumoniae (S. pneumoniae) remains a significant pathogen causing morbidity and mortality in the Southeast Asian region. A World Health Organization (WHO) 2008 bulletin indicated that the 5 of the top 10 countries with biggest numbers of pneumonia cases, namely India, China, Indonesia, Vietnam and the Philippines, are from the geographic region of the Southeast Asia. ${ }^{1}$

Invasive pneumococcal disease (IPD), where S. pneumoniae are isolated from a normally sterile site, most frequently affects children less than 2 years old, adults at least 65 years old and immunocompromised individuals. It is associated with $25 \%$ morbidity and $6 \%$ mortality rates among children and a case fatality rate of $6-24 \%$ in the same population. ${ }^{2,3}$ Mortality rates are much higher in young children in developing countries (10-40\%), likely due to poorer access to healthcare and co-morbidities. ${ }^{4}$ Currently, there are 93 known pneumococcal serotypes exhibiting a wide range of epidemiological profiles but only 20 of these account for over $80 \%$ IPD cases globally. Control measures against IPD through immunization had been directed largely against these most common serotypes causing IPD.

Vaccination has proven to be a successful intervention against infection by $S$. pneumoniae. The WHO in 2010 reports a reduction in the incidence of pneumococcal pneumonia in children less than 2 years old by about $30 \%$ since the introduction 
of the pneumococcal vaccine. ${ }^{5}$ However, it has been observed that the reduction of infection due to vaccine serotypes following immunization has been accompanied by an increase in infection due to non-vaccine serotypes. ${ }^{6-10}$

Pneumococcal conjugate vaccine 7 (PCV7), which covers $S$. pneumoniae serotypes $4,6 \mathrm{~B}, 9 \mathrm{~V}, 14,18 \mathrm{C}, 19 \mathrm{~F}$ and $23 \mathrm{~F}$ was introduced in the Philippines in 2006. ${ }^{11,12}$

It has been noted that there is scarcity of data on the serotype composition and antibiotic resistance of invasive pneuomococcal strains from developing countries. ${ }^{13}$ We describe in this study the serogroup distribution and antimicrobial resistance patterns of $S$. pneumoniae in the Philippines from 2004-2011. Each serogroup varies in prevalence, age group infected, geographical distribution, and antimicrobial resistance patterns. Knowledge of circulating serogroups and their antimicrobial susceptibility profiles is important for the development of effective vaccine strategies and will ensure a sustainable monitoring program on the effect of immunization on disease control. ${ }^{14,15}$

\section{METHODOLOGY}

\section{The isolates}

S. pneumoniae isolates from sterile body fluids were collected through the Philippine Department of Health - Antimicrobial Resistance Surveillance Program (DOH-ARSP) from January 1, 2004 to December 31, 2011. The DOH-ARSP is a sentinel based surveillance which receives isolates from 22 sentinel sites strategically distributed throughout the Philippines. ${ }^{16-19} S$. pneumoniae isolates from ARSP that were stored in skimmed milk and kept frozen at $-80^{\circ} \mathrm{C}$, and/or lyophilized were revived for re-identification. Identity of isolates were confirmed through optochin disk test and/or by bile solubility test.

\section{Antimicrobial susceptibility testing}

Antimicrobial susceptibility testing for penicillin, erythromycin, cotrimoxazole, ceftriaxone and levofloxacin was done following the Kirby Bauer Method using Clinical and Laboratory Standards Institute (CLSI) recommended antimicrobial disks and e-tests. Results were interpreted using appropriate breakpoints as defined by CLSI standards. ${ }^{20}$

\section{Typing}

Pneumococcal isolates were divided into serogroups and serotypes through slide agglutination following the Denka Seiken Slide Agglutination Method. ${ }^{21,22}$ Serogrouping through slide agglutination test has a $95.7 \%$ overall agreement with the gold standard Quellung reaction. Due to unavailability of factor sera, no further typing was done within serogroups which have several serotypes.

\section{RESULTS}

A total of 195 isolates of $S$. pneumoniae were collected from patients with IPD during the 8-year study (Table 1). The age range of the patients was $0-90$ years with a mean age of 29 years and median age of 20 years. There were more males (64\%) than females among the patients. Of the 195 isolates, 63\% (123) were from blood samples, $11 \%$ (21) were from cerebral spinal fluid and $26 \%$ (51) were from pleural fluid. Many of the isolates (39\%) were isolated from patients of the 18-64 years age group, followed by $0-6$ years old (32\%), 6-18 years old (15\%) and 65 years and older (14\%).

\begin{tabular}{|c|c|c|c|c|c|c|c|c|c|}
\hline Serotypes & 2004 & 2005 & 2006 & 2007 & 2008 & 2009 & 2010 & 2011 & Total \\
\hline 4 & & 1 & & & & 2 & 3 & 6 & 12 \\
\hline 6 & & 1 & 1 & 1 & & 1 & 7 & 4 & 15 \\
\hline 9 & & 1 & & & & & 1 & 1 & 3 \\
\hline 14 & & & & & & & 5 & 1 & 6 \\
\hline 18 & & & & & & 1 & 3 & & 5 \\
\hline 19 & & & & & & 1 & & & 1 \\
\hline 23 & & 1 & 1 & & & 1 & 4 & 1 & 8 \\
\hline 1 & 3 & 7 & 2 & 1 & 4 & 6 & 15 & 13 & 51 \\
\hline 5 & 3 & 3 & 1 & 2 & 9 & 3 & 8 & 5 & 34 \\
\hline 7 & & & & & 1 & & 1 & & 2 \\
\hline 3 & & 1 & & & & & & 6 & 7 \\
\hline 2 & & & & 1 & 2 & 2 & 1 & 1 & 7 \\
\hline 12 & & & 1 & & & 2 & 2 & 2 & 7 \\
\hline 15 & & & & & 1 & & 1 & 1 & 3 \\
\hline 17 & & & & & 1 & & & & 1 \\
\hline 20 & & & & 3 & & & & 2 & 5 \\
\hline 22 & & & 1 & & & & 1 & 2 & 4 \\
\hline 33 & & & & 1 & & & & & 1 \\
\hline 16 & & & & & & 1 & 1 & 1 & 2 \\
\hline 24 & & & & 1 & & & & & 1 \\
\hline 25 & & & & 1 & & 2 & & 1 & 4 \\
\hline 28 & & & & & & & 1 & & 1 \\
\hline 29 & & & & & & 2 & & 1 & 3 \\
\hline 31 & & & & & & & 1 & & 1 \\
\hline 34 & & & & & & & 1 & & 1 \\
\hline 38 & 1 & & & & & & 1 & & 2 \\
\hline 39 & & & & & & & 1 & & 1 \\
\hline Non-Serotypable & & & & & & 3 & 1 & 3 & 7 \\
\hline Total & 7 & 15 & 7 & 11 & 18 & 27 & 59 & 51 & 195 \\
\hline $\begin{array}{l}\text { Legend: } \\
\text { PCV7 Serotype } \\
\text { PCV10 Serotyp } \\
\text { PCV13 Serotyp } \\
\text { PPV23 Serotyp }\end{array}$ & $\begin{array}{l}14,18 \mathrm{C} \\
1,5,7 \mathrm{~F} \\
3,6 \mathrm{~A}, \\
6 \mathrm{~B}, 7 \mathrm{~F}\end{array}$ & $\mathrm{~V}, 10 \mathrm{~A}$ & 14,1 & $8 C, 19$ & $22 \mathrm{~F}, 2$ & & & & \\
\hline
\end{tabular}


Over the 8 year study period, among 195 isolates, 96\% (188) were serotyped while $4 \%$ (7) could not be typed. There were 27 serotypes identified with the most frequently occurring being serotypes 1 (26.15\%), 5 (17.44\%), 6 (7.69\%), 4 (6.15\%), 23 (4.10\%), 3 (3.59\%), 2 (3.59\%) and 12 (3.59\%) (Table 1). These 8 serotypes composed $72.3 \%$ of the total isolates.

Among patients under the age of 5 , the most common serotype were $1(13 \%) \& 6(18 \%)$ while among patients $6-17$ years and 18 64 years, serotype $1(23 \%, 43 \%)$ and $5(35 \%, 23 \%)$ were most prevalent. Among the elderly (age more than 65 years), the most frequently occurring were serotype $1(14 \%), 3(17 \%), 4(14 \%)$ and $12(14 \%)$

Coverage of currently available pneumococcal vaccines of the serogroups identified in this study were as follows: PCV7 - 25.6\%, PCV10 - 70.3\% and PCV13 - 73.8\%.

Antibiotic resistance of the pneumococcal isolates were low (Figure 1). The overall penicillin resistance were at $0 \%$ and $2.5 \%$ using the non-meningitis and meningitis CLSI breakpoints, respectively. Erythromycin resistance was $0.51 \%$, cotrimoxazole and levofloxacin resistance were at $8.20 \%$ and $1.03 \%$, respectively. All of the isolates were observed to be susceptible to ceftriaxone. Two percent of the isolated $S$. pneumoniae was observed to be multi-drug resistant (3/195).

The penicillin-resistant meningitis isolates were of serotypes 1 , 5 and 14 (Table 2). These serotypes are all covered by PCV10 and 13 but not by PCV7 which does not cover for serotypes 1 and 5 . The erythromycin resistant isolates were serotype 9 while cotrimoxazole resistant isolates were serotypes $1,5,6,12$ and 14. Forty-one percent of the cotrimoxazole resistant isolates are covered by PCV7, 88\% are covered by PCV10 and PCV13 and $6 \%$ were non-vaccine serotype. Levofloxacin resistant isolates were of serotypes 5 and 23. PCV7 coverage of levofloxacin resistant isolates were 50\% and PCV10 and PCV13 coverages were both $100 \%$.

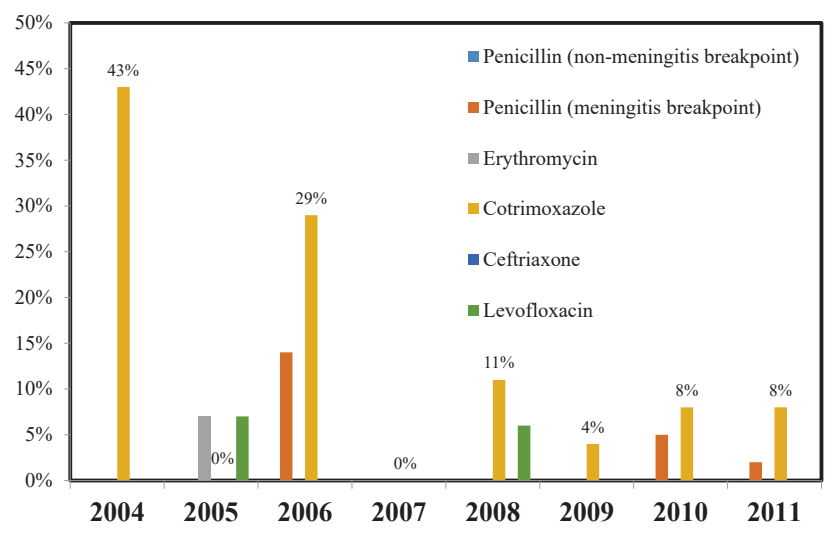

Figure 1. Resistance profile of S. pneumoniae isolates (Philippines, 2004-2011, n=195).

Table 2. Serotypes of antibiotic resistant Streptococcus pneumoniae (Philippines, 2004-2011)

\begin{tabular}{ll}
\hline Antibiotic Resistance among Streptococcus pneumoniae isolates & Serotype \\
\hline Penicillin resistance & $1,5,14$ \\
Erythromycin resistance & 9 \\
Cotrimoxazole resistance & $1,5,6,12,14$ \\
Levofloxacin resistance & 5,23 \\
\hline
\end{tabular}

\section{DISCUSSION}

\section{Serotype distribution}

The Philippines have been identified by the United Nations Children's Fund and World Health Organization to be among the 15 countries that contributes the most to the total childhood pneumonia cases worldwide registering childhood mortality rates of $37.8 / 100,000$ in year $2000 .{ }^{3}$ It is recognized that pneumococcal serotypes varies in prevalence, age group infected, geographical distribution, and antimicrobial resistance patterns. There is thus a need for country-specific knowledge of the predominant pneumococcal serotypes and their distribution. The Philippines, however, have paucity of data to provide knowledge on $S$. pneumoniae prevalence and distribution which may be used to evaluate the effect of disease prevention measures. ${ }^{23-24}$ The present study provides an estimate of the serotype distribution and antimicrobial resistance of $S$. pneumoniae causing invasive pneumococcal disease in the Philippines over an 8 year period (2004 to 2011).

The dominant serotypes associated with IPD worldwide include $14,4,1,6 \mathrm{~A}, 6 \mathrm{~B}, 3,8,7 \mathrm{~F}, 23 \mathrm{~F}, 18 \mathrm{C}, 19 \mathrm{~F}$ and $9 \mathrm{~V}$. The serotype profile seen in the present study is largely similar to the dominant IPD serotypes worldwide with the most common serotypes in this study being $1,5,6,4,23,3,2$ and 12 (Table 1). Of the 27 serotypes identified, we report here the identification of 10 serotypes - 3, 16, 17, 24, 25, 29, 31, 34, 38 and 39 - which have not been reported in previous local studies. ${ }^{23,25}$ It was noted that two nonPCV serotypes - serotypes 1 and 5 - were seen to be present yearly throughout the 8 year study period in contrast with the other serotypes which occurred sporadically over the 8-year study period. Serotype 1 were isolated yearly from age group 1964 years and were seen to occur sporadically in the rest of the age groups. Serotype 5 were consistently seen from age groups 6-18 years and 19-64 years throughout the 8 year period in the study. This observation provides support for the current local adult immunization recommendations of giving both PCV13 and polysaccharide vaccine to adults. ${ }^{26}$

Phongsamart et al. reports that in Thailand, the pneumococcal serotypes associated with invasive pneuomococcal pneumonia (IPD) among children under 5 years of age were $6 \mathrm{~B}, 6 \mathrm{a}, 9 \mathrm{~V}$, $14,19 \mathrm{~A}, 19 \mathrm{~F}$, and $23 \mathrm{~F} .{ }^{14}$ In a study done by Capeding et al. at a tertiary care center in the Philippines from 2000 to 2005, serogroup 6 was also isolated from children with IPD admitted in a tertiary hospital, and together with serotypes 18 and 14, were responsible for $50 \%$ of the admitted IPD cases in children..$^{23}$ In our study, serotype 6 was likewise the most common serotype isolated from patients under the age of 5 (18\%). Serotype 1 which was not observed from 2000-2005 in a local study was also fairly more common (13\%) in the present study.

The use of PCV7 has been reported to be successful in combating IPD cases. It is recognized that even the nonvaccinated individuals may benefit from the vaccine due to the decreased circulation of the pathogen as a result of the reduction in nasopharyngeal carriage of pneumococci among those who received the vaccine. ${ }^{27}$ There are reports, however, of increasing prevalence rate of nonPCV7 associated serotype infections following introduction of PCV7.9,27-29 According to Feiken et. al., factors that contribute to the rising cases of nonPCV7- associated serotypes include 1) secular trends in serotype prevalence occur over time, 2) changes in antibiotic use 3) characteristics 
of surveillance systems and 4) changes in susceptibility of the population to pneumococcal diseases..$^{30}$

In the present study, it was observed that the prevalence of serotypes covered by PCV7 remained stable from $27 \%$ in 2005 to $27 \%$ in 2011. Consequently, the observed prevalence of nonPCV7 serotypes likewise remained stable at $73 \%$ in 2011 which is the same nonPCV7 rate in 2004. The absence of a decrease in the prevalence of PCV7 serotypes even after the introduction of PCV7 in the country in 2006 may reflect poor coverage of PCV7 among susceptible population. Administration of PCV7 was introduced in the country in 2006 through private institutions. It was not, however, included in the mandatory free national immunization program of the government during the period of the study. Though PCV7 was available in the private institutions in the Philippines by year 2006, the vaccine is largely considered to be expensive in a generally poor and developing third-world country. Beginning the year 2013, PCV has since been included in the free national immunization program of the country. As we anticipate better PCV immunization coverage among the susceptible age groups, continued surveillance of prevailing pneumococcal serotypes may be done henceforth to monitor any shift in the prevalence of PCV associated serotypes to guide disease control measures.

\section{Antimicrobial Resistance}

The levels of antimicrobial resistance of $S$. pneumoniae isolates in many Asian countries are among the highest in the world during the early part of this century. Isolates from Vietnam showed the highest prevalence of penicillin resistance at $71.4 \%$; and erythromycin resistance at 92.1\%. Isolates from Hong Kong showed the highest rate of ciprofloxacin resistance at $11.8 \%$. Resistance to penicillin ranged from $38.6 \%$ among isolates from Taiwan to $71.4 \%$ among isolates from Vietnam. Erythromycin resistance was from $73.9 \%$ (China) to $92.1 \%$ (Vietnam) while resistance to ciprofloxacin was from $6.5 \%$ (Korea) to $11.8 \%$ (Hong Kong). Data from the Philippines showed that $18.2 \%$ of the isolates were resistant to erythromycin while $9.1 \%$ were ciprofloxacin resistant. ${ }^{32}$ Statistics from the Philippines' Antimicrobial Resistance Surveillance Program (ARSP) progress reports also showed resistance rates of pneumococci to cotrimoxazole to be higher (from 14\% in 2006 to $15 \%$ in 2011) relative to other antibiotics. ARSP annual progress reports showed that resistance rates to penicillin, and erythromycin among all pneumococcal isolates have remained low at $<4 \%$, and $2 \%$ respectively from 2007 to $2011 .^{17-19,31-32}$ The current study shows that antimicrobial resistance among IPD isolates remained low in the country over the study period (Figure 1). Resistance to cotrimoxazole was seen to decrease from $43 \%$ in 2004 to $6 \%$ in 2011 while resistance to penicillin, erythromycin and levofloxacin remained less than $5 \%$ in the later years of the study (2009-2011).

\section{Serotype and Antimicrobial Resistance}

Certain pneumococcal serotypes have been identified to be associated with specific drug resistance among pneumococcal isolates. Data would show that most penicillin-resistant and macrolide-resistant isolates are derived from five serotypes $(6 \mathrm{~B}$, $9 \mathrm{~V}, 14,19 \mathrm{~F}, 23 \mathrm{~F})$, all of which are covered by PCV7. ${ }^{4}$ In a study among 555 pneumococcal isolates from 10 Asian countries, it was observed that penicillin resistance was most common among serotypes $19 \mathrm{~F}$ and $23 \mathbf{F}$ while erythromycin resistance was more commonly seen among serotypes $19 \mathrm{~F}, 23 \mathrm{~F}, 14,6 \mathrm{~B}$ and $6 \mathrm{~A} .{ }^{33}$
An earlier study among pneumococcal isolates received at the Pneumococcal Reference Laboratory in Spain from January 1990 to December 1996 showed similar results with serotypes 6, 9, 14, 19 and 23 being associated with penicillin resistance. From the same set of isolates, it was observed that the difference in penicillin resistant rates is mainly due to differences in the prevalence of pneumococci belonging to the aforementioned serotypes. ${ }^{15}$

Contrary to data from other Asian countries, there were relatively few serogroups 19 and 23 seen in the present study and none of them were observed to be resistant to penicillin. Penicillin resistance was mostly seen among serotypes 1,5 and 14 . Half (3 out of 6 ) of the serotype 14 isolates exhibited penicillin resistance with 2 of the isolates coming from children less than 1 year. Penicillin resistance among serotype 1 was at $2 \%$ ( 1 out of 51 ) and was at $6 \%$ (2 out of 34 ) among serotype 5 . It was noted that $67 \%$ (4 out of 6 ) of penicillin resistant serotypes (1 serotype 1,1 serotype 14, and 2 serotype 5) came from age groups 6-18 years and 1964 years. Prevention of infection from pneumococcal isolates serotypes 1,5 and 14 that is conferred by both PCV10 and 13 will appear therefore to help ensure continued low resistance to penicillin among pneumococcal isolates in the country.

Eight percent of the total isolates in this study were found to be resistant to cotrimoxazole. Cotrimoxazole resistance was seen to decrease through the eight year study period from $43 \%$ in 2004 to $6 \%$ in 2011 . This reduction may reflect the shift from the use of cotrimoxazole as first line antibiotics to beta-lactam antibiotics during the study period. Cotrimoxazole resistant isolates were of serotypes $1,5,6,12$ and 14 with serotype 5 making up $44 \%$ of all isolates that were cotrimoxazole resistant and serogroup 6 accounting for $38 \%$. It was noted that there was only 1 isolate of serotype 14, the serotype associated with cotrimoxazole resistance in Asia, ${ }^{6}$ which was found to be cotrimoxazole resistant in the present study.

It was observed that $21 \%$ (7 out of 34$)$ of all serotype 5 isolates in the study were resistant to cotrimoxazole while $40 \%$ (6 out of 15) of serotype 6 were cotrimoxazole resistant. Through the eight year study period, the percentage of serotype 5 that were cotrimoxazole resistant decreased from 100\% (3 out of 3) in 2004 to $0 \%$ ( 0 out of 5 ) in 2011. In contrast, the percentage of cotrimoxazole resistance among serotype 6 increased from zero in 2005 to $50 \%$ (2 out of 4) in 2011. Cotrimoxazole resistance in the later 3 years of the study was mainly due to serotype 6 . It was also noted that $56 \%$ (9 out of 16) of cotrimoxazole resistant serotypes were seen among patients less than 5 years old and the rest were seen in the 6-17 years and 8-64 age groups. No cotrimoxazole resistant serotypes were seen among the $>65$ age groups. These observations would favor the introduction of PCV to help reduce not only the incidence of IPD among the susceptible age group but to also reduce cotrimoxazole resistance among pneumococcal isolates in the country.

Though it is not clear why particular pneumococcal serogroups have a higher probability of containing specific resistance genes, it is acknowledged that there are evidences of strong associations between resistance patterns and serotype. ${ }^{34}$ However, an exchange of the gene encoding capsular serotype can happen between pneumococcal strains through transformation. It is thus possible that highly resistant clones may become members of highly invasive serotypes which are currently not associated with drug resistance. Information from continued surveillance of 
resistant serotypes to guide immunization policies will therefore be very useful in the control of IPD as well as in the control of antimicrobial resistance among pneumococcal isolates.

To be noted in this study is the high infection rate observed in males which can be due to more exposure to socio-economically related risk factors, such as alcohol, smoking and labor in a polluted environment. ${ }^{35}$ This can also be factor in the high isolation rate of $S$. pneumoniae from the 18-59 year age group as this is generally considered as the working age group in the Philippines thus exposing the males more to the risk factors. Also, during the study period, the vaccination against $S$. pneumoniae was recommended primarily among target children or those included in 0-5 year old age group.

\section{CONCLUSION}

The present study showed that Streptococcus pneumoniae serogroup/serotype profile causing IPD in the country is largely similar to the dominant IPD serogroup/serotypes worldwide. The most common serogroups/serotypes causing IPD in this study includes 1, 5, 6, 4, 23, 3, 2 and 12. The serotype distribution of $\boldsymbol{S}$. pneumoniae in the Philippines remained stable from 2004 to 2011 and the antimicrobial resistance among the isolates remained low. The serogroups/serotypes of antibiotic resistant Streptococcus pneumoniae in this study were not similar with known serotype resistance profiles in other Asian countries. With the inclusion of PCV in the national free national immunization program of the country beginning 2013, better PCV immunization coverage among the susceptible age groups is expected. Continued surveillance of prevailing pneumococcal serotypes should be done henceforth to monitor any shift in the prevalence of PCV associated serotypes to guide disease control measures including control of emergence of resistant pneumococcal isolates.

\section{ACKNOWLEDGMENTS}

The authors thank the personnel of the sentinel sites of the Philippine Department of Health Antimicrobial Resistance Surveillance Program and the personnel of the Antimicrobial Resistance Surveillance Reference Laboratory for the contribution of their time and efforts to this study.

\section{AUTHOR DISCLOSURE}

The authors declared no conflicts of interest.

\section{FUNDING SOURCE}

None.

\section{REFERENCES}

1. Rudan I, Boschi-Pinto C, Biloglav Z, Mulholland K, Campbell H. Epidemiology and etiology of childhood pneumoniae. Bull World Health Organ. 2008;86(5):408-16. PMID: 18545744. PMCID: PMC2647437.

2. Chong CY, Koh-Cheng T, Yee-Hui M, Nancy TW. Invasive pneumococcal disease in Singapore children. Vaccine. 2008;26(27-28):3427-31. PMID: 18499309. https://doi. org/10.1016/j.vaccine.2008.04.035.

3. Bravo LC. Asian Strategic Alliance for Pneumococcal Disease Prevention (ASAP) Working Group. Overview of the disease burden of invasive pneumococcal disease in Asia.
Vaccine. 2009;27(52):7282-91. PMID: 1933708. https://doi. org/10.1016/j.vaccine.2009.04.046.

4. Lynch JP 3rd, Zhanel GG. Streptococcus pneumoniae: Epidemiology and risk factors, evolution of antimicrobial resistance, and impact of vaccines. Curr Opin Pulm Med. 2010;16(3):217-25. PMID: 20375783. https://doi.org/ 10.1097/MCP.0b013e3283385653.

5. World Health Organization. World Health Assembly 63, No. A63/26. Treatment and prevention of pneumoniae: report by the Secretariat,2010. Available from: http://apps.who.int/gb/ ebwha/pdf_files/WHA63/A63_26-en.pdf.

6. Kattan R, Rayyan AA, Zheiman I, Idkeidek S, Baraghithi S, Rishmawi N, et al. Serotype distribution and drug resistance in Streptococcus pneumoniae, Palestinian territories. Emerg Infect Dis. 2011; 17(1): 94-6. PMCID: PMC3204635. https:// doi.org/10.3201/eid1701.100886.

7. Buxbaum A, Forsthuber S, Graninger W, Georgopoulos A, Austrian Bacterial Surveillance Network. Serotype distribution and antimicrobial resistance of Streptococcus pneumoniae in Austria. J Antimicrob Chemother. 2004;54(1):247-50. PMID: 15140858. https://doi.org/10.1093/jac/dkh250.

8. World Health Organization. Index to immunization, vaccines and biologicals. Research and development. Available from: http://www.who.int/immunization/research/en/

9. Jacobs MR, Good CE, Bajaksouzian S, Windau AR. Emergence of Streptococcus pneumoniae serotypes 19A, 6C, and $22 \mathrm{~F}$ and serogroup 15 in Cleveland, Ohio, in relation to introduction of the protein-conjugated pneumococcal vaccine. Clin Infect Dis. 2008;47(11):1388-95. https://doi.org/ $10.1086 / 592972$.

10. Calatayud L, Ardanuy C, Tubau F, Rolo D, Grau I, Pallarés R, et al. Serotype and genotype replacement among macrolideresistant invasive pneumococci in adults: mechanisms of resistance and association with different transposons. J Clin Microbiol. 2010;48(4):1310-6. PMID: 20147647. PMCID: PMC2849543. https://doi.org/10.1128/JCM.01868-09.

11. Philippine Foundation for Vaccination. Childhood immunization schedule 2005. Available from: http:// www.philvaccine.org/vaccination-schedules/childhoodimmunization-schedule.

12. Philippine Foundation for Vaccination childhood immunization schedule 2006. Available from: http:// www.philvaccine.org/vaccination-schedules/childhoodimmunization-schedule.

13. Saha SK, Baqui AH, Darmstadt GL, Ruhulamin M, Hanif M, $\mathrm{El}$ Arifeen S, et al. Comparison of antibiotic resistance and serotype composition of carriage and invasive pneumococci among Bangladeshi children: implications for treatment policy and vaccine formulation. J Clin Microbiol. 2003;41(12): 55827. PMID: 14662944. PMCID: PMC308982.

14. Phongsamart W, Srifeungfung S, Dejsirilert S, Chatsuwan T, Nunthapisud P, Treerauthaweeraphong V, et al. Serotype distribution and antimicrobial susceptibility of $S$. pneumoniae causing invasive disease in Thai children younger than 5 years old, 2000-2005. Vaccine. 2007;25(7):1275-80. PMID: 17092618. https://doi.org/10.1016/j.vaccine.2006.10.001.

15. Fenoll A, Jado I, Vicioso D, Pérez A, Casal J. Evolution of Streptococcus pneumoniae serotypes and antibiotic resistance in Spain: update $(1990$ to 1996). J Clin Microbiol. 1998;36(12):3447-54. PMID: 9817852. PMCID: PMC105219.

16. Antimicrobial Resistance Surveillance Program. Antimicrobial Resistance Surveillance Program Progress Reports January December 2006, p.143. 
17. Antimicrobial Resistance Surveillance Program. Antimicrobial Resistance Surveillance Program Progress Reports January December 2007, p. 187.

18. Antimicrobial Resistance Surveillance Program. Antimicrobial Resistance Surveillance Program Progress Reports January December 2008, p. 209.

19. Antimicrobial Resistance Surveillance Program. Antimicrobial Resistance Surveillance Program Progress Reports January December 2009, p. 233.

20. Clinical and Laboratory Standards Institute. Performance standards for antimicrobial susceptibility testing; Twenty-first informational supplement. CLSI document M100S21. Wayne (PA): Clinical and Laboratory Standards Institute, 2015.

21. Shutt CK, Samore M, Carroll KC. Comparison of Denka Seiken Slide Agglutination Method to the Quellung Test for Serogrouping of Streptococcus pneumoniae Isolates. J Clin Microbiol. 2004;42(3):1274-6. PMCID: PMC356897. https:// doi.org/ 10.1128/JCM.42.3.1274-1276.2003.

22. Denka Seiken Co., Ltd. Bacterial Typing Antisera Handbook, 2006, p.41.

23. Capeding MRZ, Sombrero LT, Esparar GA, Mondoy MU, Taclibon AG. Pneumococcal serotypes among Filipino children admitted in a tertiary care center for infectious diseases from 2000 to 2005. Pediatr Infect Dis Soc Philipp J. 2009; 10(1):2-4.

24. Lucero MG, Nohynek H, Williams G, Tallo V, Simões EA, Lupisan S, et al. Efficacy of an 11-valent pneumococcal conjugate vaccine against radiologically confirmed pneumonia among children less than 2 years of age in the Philippines: a randomized, double-blind, placebo-controlled trial. Pediatr Infect Dis J. 2009;28(6):455-62. PMID: 19483514. https://doi. org/ 10.1097/INF.0b013e31819637af.

25. Capeding MRZ, Sombrero LT, Lucero MG, Saniel MC. Serotype distribution and antimicrobial resistance of invasive Streptococcus pneumoniae isolates in Filipino children. J Infect Dis. 1994;169(2):479-80.

26. Philippine Foundation for Vaccination. Schedule for adult immunization 2015. Available from: http://www.philvaccine. org/vaccination-schedules/adult-immunization-schedule

27. Shen CF, Wang SM, Lee KH, Ho TS, Liu CC. Childhood invasive pneumococcal disease caused by non-7-valent pneumococcal vaccine (PCV7) serotypes under partial immunization in Taiwan. J Formos Med Assoc. 2013;112(9):561-8. PMID: 23916313. https://doi. org/10.1016/j.jfma.2013.05.015.
28. Tsai HY, Chen YH, Liao CH, Lu PL, Huang CH, Lu CT, et al. Trends in the antimicrobial susceptibilities and serotypes of Streptococcus pneumoniae: results from the Tigecycline in vitro surveillance in Taiwan (TIST) study, 2006-2010. Int J Antimicrob Agents. 2013;42(4):312-6. PMID: 23849332. https://doi.org/10.1016/j.jjantimicag.2013.05.013.

29. Huang SS, Platt R, Rifas-Shiman SL, Pelton SI, Goldmann D, Finkelstein JA. Post-PCV7 changes in colonizing pneumococcal serotypes in 16 Massachusetts communities, 2001 and 2004. Pediatrics. 2005;116(3):e408-13. PMID: 16140686. https://doi.org/10.1542/peds.2004-2338.

30. Feikin DR, Kagucia EW, Loo JD, Link-Gelles R, Puhan MA, Cherian T, et al. Serotypespecific changes in invasive pneumococcal disease after pneumococcal conjugate vaccine introduction: a pooled analysis of multiple surveillance sites. PLoS Med. 2013;10(9):e1001517. PMID: 24086113. PMCID: PMC3782411. https://doi.org/10.1371/journal. pmed.1001517.

31. Antimicrobial Resistance Surveillance Program. Antimicrobial Resistance Surveillance Program Progress Reports January December 2010, p. 251.

32. Antimicrobial Resistance Surveillance Program. Antimicrobial Resistance Surveillance Program Progress Reports January December 2011, p. 267.

33. Song JH, Jung SI, Ko KS, Kim NY, Son JS, Chang HH, et al. High prevalence of antimicrobial resistance among clinical Streptococcus pneumoniae isolates in Asia (an ANSORP study). Antimicrob Agents Chemother. 2004;48(6):21017. PMID: 15155207. PMCID: PMC415617. https://doi. org/10.1128/AAC.48.6.2101-2107.2004.

34. Schrag SJ, Beall B, Dowell S. Resistant pneumococcal infections: the burden of disease and challenges in monitoring and controlling antimicrobial resistance. Switzerland: World Health Organization,2001. Available from: http://www.who. int/drugresistance/technicalguidance/en/resistantinfection.pdf.

35. Molander V, Elisson C, Balaji V, Backhaus E, John J, Vargheese $\mathrm{R}$, et al. Invasive pneumococcal infections in Vellore, India: clinical characteristics and distribution of serotypes. BMC Infect Dis. 2013;13:532. PMID: 24206667. PMCID: PMC3827497. https://doi.org/10.1186/1471-2334$13-532$.

Disclaimer: This journal is OPEN ACCESS, providing immediate access to its content on the principle that making research freely available to the public supports a greater global exchange of knowledge. As a requirement for submission to the PJP, all authors have accomplished an AUTHOR FORM, which declares that the ICMJE criteria for authorship have been met by each author listed, that the article represents original material, has not been published, accepted for publication in other journals, or concurrently submitted to other journals, and that all funding and conflicts of interest have been declared. Consent forms have been secured for the publication of information about patients or cases; otherwise, authors have declared that all means have been exhausted for securing consent. 